TIPA. Travaux interdisciplinaires sur la parole et le langage

$35 \mid 2019$

Emo-langages

\title{
Langues, arts et empathie à l'école
}

Communauté et singularité d'expérience

Languages, arts and empathy at school: community and singularity of

experience

\section{Marie Potapushkina-Delfosse}

\section{OpenEdition \\ Journals}

Édition électronique

URL : http://journals.openedition.org/tipa/3375

DOI : 10.4000/tipa.3375

ISSN : 2264-7082

Éditeur

Laboratoire Parole et Langage

Référence électronique

Marie Potapushkina-Delfosse, "Langues, arts et empathie à l'école», TIPA. Travaux interdisciplinaires sur la parole et le langage [En ligne], 35 | 2019, mis en ligne le 23 juillet 2019, consulté le 25 juillet 2019 URL : http://journals.openedition.org/tipa/3375 ; DOI : 10.4000/tipa.3375

Ce document a été généré automatiquement le 25 juillet 2019.

\section{(c) (i) (9)}

La revue TIPA. Travaux interdisciplinaires sur la parole et le langage est mise à disposition selon les termes de la licence Creative Commons Attribution - Pas d'Utilisation Commerciale - Pas de Modification 4.0 International. 


\title{
Langues, arts et empathie à l'école
}

\author{
Communauté et singularité d'expérience \\ Languages, arts and empathy at school: community and singularity of \\ experience
}

Marie Potapushkina-Delfosse

\section{Introduction}

1 Dans nos cultures occidentales enclines à dissocier le mental et l'affectif et à considérer l'apprentissage comme un exercice purement cérébral d'accumulation de savoirs, des changements s'orientant vers une compréhension plus nuancée de la nature de la connaissance ont commencé à s'opérer dès les années 1930, notamment grâce à la réflexion de J. Dewey qui envisageait les processus cognitifs comme étant inséparables du domaine $\mathrm{du}$ sensible. Un dispositif d'enseignement/apprentissage d'une langue étrangère, basé sur des pratiques artistiques, élaboré à partir des travaux de Dewey et mis en œuvre dans une classe d'école primaire, fait l'objet de cet article. Les bénéfices que les élèves tirent de l'accès aux langues par les arts en termes de développement de compétences langagières (mémorisation lexicale, maîtrise de la syntaxe, qualité de la prononciation) ayant été mis en évidence précédemment (Potapushkina-Delfosse, 2014, 2016), dans le présent article je m'intéresse aux liens émotionnels tissés au cours de l'expérimentation, en particulier aux rapports d'empathie que les élèves ont pu établir entre eux, envers les personnages des œuvres étudiées ainsi qu'à l'égard des adultes investis dans le projet.

2 Dans un premier temps, le lecteur de cet article sera invité à se familiariser avec la définition de l'expérience aboutie, donnée par Dewey, ainsi qu'avec les raisons pour lesquelles elle est atteignable par la pratique et/ou au contact de l'art. Nous verrons ensuite que l'art partage un certain nombre de caractéristiques avec l'empathie, concept qui sera élucidé à partir de travaux en philosophie (phénoménologie), en neuropsychologie et en neurosciences affectives et développementales, consacrés à l'empathie au cours de deux dernières décennies. Après la présentation du cadre 
méthodologique de la recherche (structure et contenus du dispositif didactique, méthodologie adoptée pour le recueil et le traitement de données), seront analysés quelques extraits représentatifs du corpus, illustrant diverses attitudes empathiques développées par les élèves. Enfin, seront exposés les résultats de cette recherche qualitative qui, malgré ses limites, ouvre de nouvelles perspectives à explorer en didactique des langues.

\section{Cadre théorique : deux modes de connaissance apparentés}

\subsection{De l'art à l'empathie}

3 Dans le domaine de la didactique des langues-cultures, des dispositifs expérientiels commencent à être élaborés et testés à différents niveaux d'enseignement scolaire (Aden, 2017 ; Eschenauer \& Voise, 2017; Potapushkina-Delfosse, 2018). Ces travaux s'inscrivent dans une perspective où le savoir émerge d'une expérience plurielle (cognitive, affective, sociale, culturelle) vécue par l'élève accueilli comme un être global, en dehors de cloisonnements disciplinaires habituels.

4 Afin de clarifier le concept de dispositif expérientiel, tournons-nous vers Dewey. Il définit l'expérience comme "l'interaction de l'être vivant et de son environnement (qui) fait partie du processus même de l'existence» $(2014: 80)$ et distingue deux types d'expérience :

- tronquée, diffuse (effectuée par un sujet plus ou moins distrait, généralement non menée à terme) :

- aboutie (conduite par un sujet qui accorde l'intégralité de son attention à la tâche réalisée ; processus harmonieux dont le résultat et surtout le déroulement apporte satisfaction à son auteur).

Qu'elle soit tronquée ou aboutie, toute expérience est empreinte d'émotions: cette intuition de Dewey est confirmée par Damasio, pour qui elles constituent « des ensembles compliqués de réponses chimiques et neuronales» (2002: 59) et "font partie des dispositifs biorégulateurs dont nous sommes équipés pour survivre» (Ibid.: 61). L'expérience tronquée est caractérisée, selon Dewey, par des séries d'émotions proches de réflexes qui déclenchent ou ponctuent le processus expérientiel mais n'évoluent pas : soit l'émotion est trop insignifiante pour être saisie soit tellement puissante qu'elle envahit la conscience du sujet et inhibe l'action entamée. L'expérience aboutie, quant à elle, est sous-tendue par des émotions qui ne provoquent pas d'explosions directes (éclat de rire, coups de poings, etc.) mais, façonnées par une résistance émanant du sujet, propulsent, sélectionnent, agencent une expérience. La notion de résistance, évoquée par Dewey et reprise par Damasio, n'est autre que la raison qui, d'un côté, canalise le flux émotionnel, le tient sous contrôle et, de l'autre, a besoin de l'émotion comme source et moteur.

6 Les dispositifs didactiques que l'on qualifie d'expérientiels visent à emmener les apprenants à des expériences abouties. Celles-ci pouvant résulter, selon Dewey, d'activités de natures diverses, se manifestent toutefois le plus aisément dans la pratique et le contact des arts. Ce terrain est particulièrement favorable à la mise en œuvre de la caractéristique principale d'une expérience aboutie, à savoir, la simultanéité de l'action et de la perception, accompagnée d'une conscientisation des liens entre ce qui est fait et ce 
qui est subi, entre l'individu et l'environnement qui agissent simultanément l'un sur l'autre (dans la partie de l'article, dédiée à l'analyse du corpus, je me référerai à ce mécanisme en employant le terme « équilibre perception/action »).

7 Contrairement à une vision commune qui qualifie de perceptif le comportement de l'individu en contact avec l'art (auditeur, spectateur, lecteur...) et d'actif le comportement de l'artiste, Dewey note la nécessité de la coexistence des deux caractéristiques dans les deux postures. Le récepteur d'une œuvre d'art, qui traverse une expérience aboutie déclenchée par celle-ci, dépasse l'état de perception pour co-agir/co-créer avec l'auteur de cette œuvre. Quant à l'artiste, il n'agit jamais sans percevoir. Ces deux postures (celles de l'artiste et du public) à la fois perceptives et actives impliquent une attitude empathique. Ainsi, le spectateur qui vit une expérience esthétique entre dans un rapport empathique avec l'auteur, l'interprète ou les personnages de l'œuvre explorée. Pour ce qui est de l'artiste, même en œuvrant dans la solitude, il entretient un rapport d'empathie avec un interlocuteur virtuel.

Il n'est donc pas surprenant que l'empathie et l'art bénéficient de définitions semblables :

- «l'empathie est un mode de connaissance» (Pacherie, 2004 : 150) «non seulement d'autrui mais aussi du monde et de nous-mêmes " (Ibid. : 152) ;

- «l'art est un mode de la connaissance » (Dewey, 2014 : 465), l'idée répandue en philosophie depuis l'antiquité (Aristote) que Dewey partage et développe :

(...) tant dans la production que dans la jouissance perceptive des œuvres d'art, la connaissance est transformée; elle devient quelque chose de plus qu'une connaissance dans la mesure où elle fusionne avec des éléments non intellectuels pour former une expérience qui vaut d'être vécue (Ibid. : 467).

9 L'art et l'empathie apparaissant comme instruments de connaissance, on peut émettre l'hypothèse qu'ils constitueront une base adéquate d'enseignements/apprentissages scolaires, notamment dans le domaine des langues vivantes étrangères (LVE). En effet, chaque nouveau langage est construit avec appui sur des langages familiers. L'art qui est «la forme de communication la plus universelle et la plus libre» (Ibid. : 440) se trouve «relégué aux musées» (Ibid.) dans le monde des adultes mais fait partie de la vie quotidienne des enfants qui ont recours aux pratiques relevant des langages artistiques (narration-fiction, dessin, chant, etc.) afin de s'exprimer et de communiquer au même titre (voire plus) qu'au langage verbal "ordinaire». Cela autorise à supposer que ces langages seront des médiums appropriés pour conduire l'enfant à l'adoption d'un nouveau langage verbal (LVE). En mettant en œuvre des dispositifs d'enseignement de l'anglais au travers des arts depuis plusieurs années, j'ai pu montrer qu'une telle approche contribue au développement de compétences langagières spécifiques. Facilite-telle l'acquisition de la compétence transversale d'empathie ? Le présent article apportera des éléments de réponse à cette question.

\subsection{Mécanismes de l'empathie}

10 L'empathie est une « simulation (...) de la subjectivité d'autrui » (Berthoz, Jorland, 2004: 10) qui est loin d'être une simple projection intellectuelle dans ce que l'autre peut ressentir ou penser. Le corps étant «le point zéro de toute orientation » (définition husserlienne rappelée par Depraz, 2004: 187), l'empathie en tant que mouvement psychique n'est possible que dans la spatialité particulière de notre corps vécu et dans un couplage temporel avec le corps de l'autre. L'empathie est semblable à un voyage: on 
quitte momentanément notre « chez soi » mental et émotionnel pour s'aventurer dans les chemins de la pensée et des affects d'autrui, comparables aux "chemins dans l'espace » (Berthoz, 2004: 254), ce qui constitue le contraire de la visée unique propre à l'être égocentré. Or, le jeune enfant en est un de par son organisation psychique. Berthoz rappelle la théorie de Piaget : avant 7-8 ans, «l'enfant (est) prisonnier de ses schémas mentaux et comme une personne enfermée dans un labyrinthe avec un seul chemin conduisant à une sortie unique, une seule vision (au sens de "visée » selon Husserl) du monde » (Ibid. : 254). A partir de l'âge de 7-8 ans, l'enfant est capable de passer d'un traitement égocentré à une perspective allocentrée (Ibid. : 255) : il s'agit d'un potentiel qui est développé à différents degrés selon les caractéristiques individuelles de chaque enfant et l'éducation dont il bénéficie. En France, Le socle commun de compétences, de connaissances et de culture exige que l'école forme les futurs citoyens à « être capables de faire preuve d'empathie et de bienveillance » (Ministère de l'Education nationale, $2015: 5$ ). Au-delà de déclarations politiques, l'empathie est explicitement enseignée dans les écoles danoises. Une attention particulière lui est portée au sein du système éducatif en Finlande : une étude longitudinale menée sur dix ans dans de nombreuses écoles de ce pays (Siekkinen et al., 2013) a montré qu'un climat scolaire empreint d'empathie contribuait à faire disparaître les phénomènes d'exclusion sociale, à augmenter la confiance des élèves en leurs capacités ainsi qu'à améliorer leurs résultats scolaires, ces derniers étant plus dépendants du climat de sécurité émotionnelle que des facteurs matériels tels que le nombre d'élèves par classe, la qualité des équipements etc.

11 Comme toute compétence scolaire ou sociale, celle d'empathie possède différents niveaux de complexité, qui sont atteints progressivement. Ainsi E. Pacherie (2004: 166) établit trois degrés d'empathie, il s'agit de la compréhension:

1. du type de l'état affectif d'autrui ;

2. du type et de l'objet de cet état ;

3. du type, de l'objet et des raisons de cet état.

12 Afin de parvenir au premier degré d'empathie, l'individu procède généralement par perception directe, comme l'explique Pacherie (Ibid.) Ainsi, un bébé qui ne saurait nommer l'émotion éprouvée par la personne se trouvant en face de lui, peut imiter son expression faciale et saisir les équivalences entre les transformations corporelles qu'il voit chez la personne en question et les effets proprioceptifs provoqués par l'imitation de cette expression. Quel que soit notre âge, nous sommes tous assujettis à ce mécanisme que l'on appelle résonnance motrice : «l'observation d'une action accomplie par autrui évoque automatiquement chez l'observateur une représentation motrice de l'action observée à condition que celle-ci appartienne à son répertoire comportemental » (Ibid. : 169). Le premier degré d'empathie, issu de ce phénomène, relève donc de dispositions innées et demeure non intentionnel et peu contrôlable. Quant au second et au troisième degrés d'empathie, nous y parvenons par le biais de «l'imagination et de la simulation projective»(Ibid.: 167), plus intentionnelle et contrôlée: nous nous mettons, en imagination, à la place de l'autre et projetons sur lui, avec plus ou moins d'ajustements, notre propre expérience, notre propre façon de raisonner. Un effort d'ajustement important est requis pour comprendre les émotions d'une personne dont la culture diffère considérablement de la nôtre, d'un porteur d'un handicap mental, d'un enfant quand on est adulte et, inversement, de l'adulte quand on est enfant. Accomplir cet effort équivaut à construire un « cadre de référence interne d'autrui » (Decety, 2004 : 59), sans 
lui substituer le nôtre, ce qui caractérise un acte d'empathie abouti. Celui-ci englobe les quatre aspects suivants, définis par Reik, en 1948 et confirmés par Decety (Ibid. : 58) :

- incorporation (accordage avec la corporéité de l'interlocuteur, moyennant la résonance motrice face à ses mimiques faciales, ses gestes, ses attitudes) ;

- identification (immersion dans l'univers mental de l'interlocuteur, la distance entre «toi » et « moi » s'amenuisant sans jamais disparaître) ;

- réverbération (le sujet éprouve les sensations et les émotions de son interlocuteur et lui communique sa compréhension de celles-ci en retour);

- détachement (malgré la proximité affective avec l'interlocuteur, le sujet ne se soumet pas à une contagion émotionnelle et ne perd jamais de vue la condition du «comme si »; il est apte à porter un regard réflexif sur son état d'empathie envers autrui et à s'extraire de cet état quand il en ressent le besoin).

13 Ce tour rapide de la littérature permet de réaliser que l'empathie est un mécanisme multiforme et complexe. Jusqu'à quel degré de jeunes élèves sauront-ils le développer? Lesquels de ses aspects seront-ils capables d'embrasser? L'analyse du corpus constitué d'entretiens menés avec les élèves, dont quelques extraits seront présentés plus loin, tentera de répondre à ces questions.

\section{Cadre méthodologique : produire une action pédagogique et analyser son impact}

\subsection{Structure et contenus du dispositif}

Il s'agit d'une recherche action qui est effectuée dans l'action (la chercheuse est impliquée en tant qu'enseignante), par l'action (le projet pédagogique conduit auprès d'élèves permet le recueil de données nécessaires à l'analyse) et pour l'action (la recherche vise à optimiser l'action pédagogique future).

L'expérimentation a été menée tout au long d'une année scolaire dans une école des Hauts-de-Seine, au sein d'une classe de CE1 (26 élèves : 14 filles et 12 garçons, âgés de 7 à 8 ans), socio-culturellement hétérogène, dont j'avais la responsabilité en tant que professeur des écoles.

16 Le dispositif didactique servant de base à l'étude est conçu dans l'intention de faire entrer les élèves dans une nouvelle langue en instaurant des liens d'empathie (ce qui est une condition indispensable de la co-création) avec tous les partenaires, fictifs et réels, côtoyés au cours du processus : les personnages des œuvres abordées, les artistes (auteurs de ces œuvres et artistes rencontrés dans le cadre du projet), les pairs.

Chez un spectateur adulte, la co-création avec l'auteur est le plus souvent invisible : il cocrée mentalement. Or, pour apprendre ce procédé, l'enfant a besoin de le vivre de façon active. C'est cette possibilité que j'ai souhaité donner aux élèves en les engageant dans un dispositif annuel composé des quatre phases suivantes :

- création picturale, gestuelle, verbale (en français) autour des œuvres de S. Delaunay; transposition d'éléments verbaux en anglais; apprentissage d'un texte en anglais ainsi cocréé à l'aide du geste pictural et dansé ;

- création verbale (en français) autour de la légende Le joueur de flûte de Hamelin; apprentissage d'un texte adapté en anglais au travers d'une gestuelle corporelle et picturale élaborée collectivement ; 
- création gestuelle (inspirée par la pédagogie théâtrale de Lecoq, 1997) autour du conte Le pinceau magique ; apprentissage du texte adapté en anglais par le jeu théâtral ;

- création d'une version gestuelle du poème The Tyger de W. Blake et l'apprentissage du texte moyennant cette version ; transposition dansée du poème, guidée par la compagnie de danse Mobilis Immobilis, avec une représentation en fin d'année. œuvres littéraires à thématiques analogues et d'origines variées ont été découvertes en français. Pendant l'année, de nombreux artistes peintres du $19^{\text {ème }}$ et du $20^{\text {ème }}$ siècles ont été présentés aux élèves à travers les contes dont la trame était basée sur une série d'œuvres de chaque artiste concerné et accompagnée musicalement.

19 A la fin du projet, je me suis entretenue avec l'ensemble des élèves de la classe afin de découvrir leur vision de l'expérience vécue au cours de l'année. Quel type d'entretien choisir? Les entretiens dirigés ou semi-dirigés ne constituent pas une forme adéquate pour collecter la parole des enfants de 7 à 8 ans, dans le cadre d'une recherche comme la nôtre. En effet, le questionnement, tel qu'il est pratiqué dans ces types d'entretiens, met les enfants dans une situation qu'ils perçoivent comme institutionnelle. Les plus timides donnent alors des réponses courtes et peu signifiantes. Les plus « scolaires " s'évertuent à trouver des réponses « justes ", comme s'ils passaient un test. Les plus sensibles, quant à eux, essaient de dire ce qui, à leur avis, fera plaisir à la personne qui les interroge : rappelons que, dans notre cas, l'intervieweuse est l'enseignante de la classe et que les enfants de l'âge concerné ont tendance à forger des liens affectifs forts avec leur professeur. Comme le montre mon expérience accumulée durant plusieurs années de recherche action à l'école primaire, les réponses ainsi obtenues sont orientées et ne permettent pas d'accéder à ce que les enfants ressentent et pensent vraiment.

L'entretien d'explicitation (Vermersch, 2010), qui vise à susciter la "parole incarnée " (Varela, Thompson, Rosch, 1993), semble plus adapté. Cependant, sa méthodologie établie pour accueillir la parole d'adultes, nécessite des adaptations lorsque les interviewés sont des enfants. La personne qui passe un entretien d'explicitation est invitée à se plonger dans le souvenir d'une action réelle qu'elle a effectuée, de la revivre, dans son imagination, en verbalisant avec le plus de précision possible chacune de ses étapes, sur les plans matériel et sensoriel. Or, l'immersion purement mentale dans une expérience vécue antérieurement est une tâche trop abstraite pour un enfant de 7-8 ans. Afin de transporter cette expérience dans l'objectivité du présent, il a besoin d'une médiation. Celle que j'ai choisie consistait à demander à chaque enfant de dessiner un ou plusieurs épisodes de son année d'anglais et d'arts avant de procéder à la verbalisation. Le dessin en tant que «narration graphique » (Luquet, $1984: 160$ ), qui requiert des efforts moteurs et émotionnels, emmène l'enfant à se concentrer entièrement sur le vécu revisité, sans se laisser distraire par des facteurs extérieurs.

21 La technique d'explicitation préconise des entretiens individuels : un intervieweur adulte accueille les propos d'un interviewé adulte, en demandant des précisions supplémentaires sur certains points, en relançant le récit lorsque celui-ci s'essouffle, etc. Face au public d'enfants, j'ai opté pour une forme collective (groupes de deux à trois élèves, formés par affinité). Pour expliquer ce choix, notons que certains enfants, lorsqu'ils s'adressent à un adulte, surtout dans une situation comme la nôtre (existence d'un rapport formel élève/enseignant), tendent à adapter leur récit, ce qui le rend moins riche. D'autres enfants, à l'inverse, restent spontanés dans leur manière de s'exprimer, en parlant à l'adulte comme s'ils parlaient à leurs pairs. Cela pose également problème car 
l'interviewer adulte n'est pas à même de déchiffrer le code enfantin dans toutes ses nuances et risque de ne pas toujours interpréter avec justesse ce qu'il entend, à ne pas rebondir au moment opportun, à passer à côté d'une possibilité de faire élucider un détail qui compte. Alors, il a été proposé à chaque enfant d'expliciter son dessin à un(e)/des camarade(s), ce(s) dernier(s) étant encouragé(s) à poser des questions pour comprendre le récit le mieux possible. Chaque enfant s'est donc trouvé alternativement dans les rôles de celui qui explicite et de celui qui écoute et questionne. Cette organisation a remédié à l'asymétrie (interviewer adulte/interviewé enfant) qui aurait pu entraver l'authenticité des échanges dans le cadre d'un entretien individuel. Les événements relatés par le narrateur éveillaient immanquablement des souvenirs chez l'auditeur, une discussion s'engageait alors entre les enfants en leur faisant presque oublier ma présence. Chacun était motivé à persévérer dans la remémoration de ses actions, ses émotions, ses sensations, ses pensées survenues au moment évoqué, tout en se reliant à l'expérience de l'autre. Cette forme d'entretien a ainsi permis aux enfants non seulement de retrouver le souvenir des états d'empathie passés mais aussi d'en vivre de nouveaux in situ.

Les transcriptions de ces entretiens, qui ont duré de 15 à 30 minutes en fonction des groupes, ont fait l'objet d'une analyse de discours, qui a rendu possible l'identification de divers éléments se rapportant aux états d'empathie éprouvés par les élèves.

Tout au long de l'année, j'ai tenu un journal de bord où je consignais avec soin mes observations quant à la manifestation de l'empathie par les élèves envers leurs pairs, les personnages des œuvres étudiées et les adultes engagés dans le processus éducatif, notamment les artistes. Je me suis également entretenue avec les artistes qui ont participé au projet (entretiens individuels semi-dirigés cette fois-ci) en les invitant à livrer leurs observations en ce qui concernait les manifestations de l'empathie chez les élèves au cours des ateliers qu'ils avaient animés. En analysant les entretiens menés avec les élèves, j'ai croisé mon interprétation des propos recueillis avec ces observations (parole des artistes et mon journal de bord) pour le maximum d'objectivité, une telle triangulation de données étant préconisée par Raby $(2008: 13)$ et Aden $(2012: 17)$ pour la recherche qualitative en didactique des langues.

\subsection{Fuvres supports du dispositif}

24 Avant de nous intéresser à l'analyse du corpus, il convient d'exposer les raisons du choix des œuvres supports en termes de relations qu'elles entretiennent avec le concept d'empathie.

Les œuvres choisies de S. Delaunay (La Prose du Transsibérien et de la Petite Jehanne de France, co-créée avec B. Cendrars, en 1913, et la série de Rythmes colorés, produite dans les années 1950) ont été réalisées en résonnance aux travaux d'autres artistes s'exprimant dans d'autres langages artistiques que la peinture : l'écriture littéraire, dans le premier cas, et la musique (jazz) dans le second. Delaunay investit différents aspects de la relation empathique (Decety, 2004: 58) envers ses confrères. Elle procède à l'identification et à l'incorporation lorsqu'elle se projette dans les œuvres en question jusqu'à en appréhender le processus de création, pour les compositions de jazz, et à rejoindre Cendrars dans son élan créatif, pour ce qui est du travail sur La Prose du Transsibérien. La réverbération a lieu quand Delaunay transpose l'expérience créative partagée dans le langage qui lui est propre: celui de la peinture. Le détachement se révèle par le fait que 
les œuvres picturales réalisées ainsi sont parfaitement autonomes et peuvent être appréciées par le public sans que ce dernier connaisse leurs sources exogènes.

Le Joueur de flûte de Hamelin est une légende médiévale allemande qui relate une catastrophe sans précédent endurée par la ville de Hamelin : des rats envahissent la cité et, après y avoir dévoré toutes les denrées alimentaires, commencent à s'attaquer aux habitants. Un musicien inconnu emploie la puissance de son art pour faire sortir les rats de la cité. Il se livre à un mouvement empathique double : vis-à-vis des citadins et envers les animaux, en reconstituant « le cadre de référence interne » (Decety, 2004:59) des uns et des autres, dont chacun est très éloigné de sa propre façon de percevoir le monde. L'artiste-magicien, ne partageant ni les valeurs des bourgeois ni les instincts des rats, accomplit, dans les deux cas, un acte d'empathie nécessitant une décentration importante. S'il utilise la compréhension de l'état affectif et mental des bourgeois pour les secourir, il emploie la connaissance de celui des rongeurs afin de mener ces derniers à leur perte (notons que l'empathie n'engendre pas toujours des actes bienveillants; elle est parfois à l'origine de comportements nuisibles, comme l'explique N. Catheline dans ses travaux sur le harcèlement, 2015). N'ayant pas obtenu de récompense promise par les notables, le flûtiste a de nouveau recours à ses pouvoirs mais, cette fois-ci, pour punir et non pour aider les citadins : il fait sortir de la ville tous les enfants et les enferme dans une grotte. Avant de partir, il jette sa flûte par terre. Celle-ci sera récupérée par un enfant retardataire qui ne s'est pas fait enfermer avec les autres. Il découvre l'instrument, l'essaye et parvient à trouver le son qui lui permettra de libérer ses camarades. Ces éléments de la trame se référent symboliquement à la transmission ainsi qu'à l'expérience personnelle dans l'apprentissage d'une pratique artistique, à la transcendance de l'art ainsi qu'à sa fonction sociale.

Le conte chinois traditionnel Le pinceau magique raconte l'histoire d'un jeune homme pauvre qui rêve de devenir peintre calligraphe mais n'en a pas les moyens. Un matin, au réveil, il trouve sur son lit un pinceau magique : tout ce que le jeune homme peindra à l'aide de ce pinceau prendra vie. Il met ce don au service de ses concitoyens, en dessinant de l'eau pour étancher la soif des uns, de la nourriture pour assouvir la faim des autres. L'empathie est utilisée ici à des fins altruistes et l'art, encore plus que dans le texte précédent, est investi d'une mission sociale, les motifs esthétiques du peintre allant de pair avec les motifs éthiques. Lorsque l'empereur s'empare du pinceau, avec l'intention de multiplier ses richesses déjà nombreuses, il est puni pour sa cupidité (forme d'égocentrisme empêchant le mouvement empathique).

Le poème The Tyger de W. Blake (1794) a été choisi pour la beauté de ses métaphores donnant libre cours à l'imagination des enfants ainsi que pour sa rythmicité qui se prête à une transposition dansée. La découverte et l'apprentissage, en anglais, de quelques strophes de ce poème, ont été accompagnés par la lecture, en français, de l'album de $\mathrm{C}$. J. Hong Le Prince Tigre (2005). L'histoire met en scène une tigresse qui pleure la perte de ses petits tués par des chasseurs. Emplie de haine et de chagrin, elle ravage le pays. Le roi se prépare à lever son armée contre elle, mais une vieille femme qui sait prédire l'avenir le lui déconseille. Selon elle, la colère de la tigresse ne sera apaisée que si le roi lui envoie son fils. Désespéré par l'idée de perdre son enfant mais contraint à sauver le pays, le roi laisse le garçon à l'entrée de la forêt. Quand la tigresse s'approche de l'enfant, elle le prend dans sa gueule pour le dévorer, mais soudain ce geste lui rappelle ses petits : c'est ainsi qu'elle les portait et les caressait. Cette attitude corporelle retrouvée déclenche chez le personnage un état affectif qui se traduit d'abord par la compassion (forme suprême 
d'empathie: Depraz, 2004), puis par l'amour maternel. A la fin de l'histoire, le prince élevé par la tigresse retrouve ses parents. Il acceptera sa destinée humaine sans toutefois renoncer à son passé animal, en confiant l'éducation de son propre fils à la tigresse, sa mère adoptive.

Certaines de ces œuvres interrogeant la nature de la création artistique et toutes mettant en lumière des comportements empathiques, sont susceptibles, selon mon hypothèse, à servir de supports appropriés au dispositif qui fait l'objet de la présente recherche.

\section{Analyse d'éléments de corpus choisis}

\subsection{Attitude empathique envers un personnage fictif}

Les trois types d'attitude empathique recherchés (à l'égard des personnages, des pairs et des adultes) ont été identifiés au cours de l'analyse des entretiens conduits auprès des élèves, sous forme d'évocation d'un souvenir (empathie ressentie dans le passé) et, pour les deux premiers types seulement, sous forme d'expression d'un état présent éprouvé lors de l'échange, en situation d'entretien. Chacun de 26 élèves a manifesté au moins un type d'attitude empathique.

\begin{tabular}{|l|l|}
\hline Type d'attitude empathique & $\begin{array}{l}\text { Nombre d'élèves ayant manifesté ce } \\
\text { type d'attitude }\end{array}$ \\
\hline Attitude empathique envers un personnage fictif & 7 \\
\hline $\begin{array}{l}\text { Souvenir d'une attitude empathique envers un } \\
\text { personnage fictif }\end{array}$ & 20 \\
\hline $\begin{array}{l}\text { Attitude empathique envers un homologue (entre } \\
\text { pairs) }\end{array}$ & 15 \\
\hline $\begin{array}{l}\text { Souvenir d'une attitude empathique envers un } \\
\text { homologue (entre pairs) }\end{array}$ & 9 \\
\hline $\begin{array}{l}\text { Souvenir d'une attitude empathique envers un } \\
\text { artiste }\end{array}$ & 8 \\
\hline
\end{tabular}

Penchons-nous tout d'abord sur l'empathie éprouvée à l'égard de personnages fictifs.

Comme l'indique Bettelheim (1976), l'enfant s'identifie aux personnages de contes, ce qui l'aide à trouver des solutions à ses préoccupations actuelles, à se préparer aux épreuves futures, à affronter la complexité de la vie.

Lors des entretiens, une élève énumère les personnages qui l'ont particulièrement marquée en illustrant chacun par un geste caractéristique (le boitement de l'enfant qui a hérité de la flûte magique; des mouvements du pinceau manipulé par l'artiste issu du conte chinois...) Trois autres élèves miment les actions clés des personnages en restituant les trames des contes qu'ils ont préférés : deux en mettant en jeu leur corps entier et le troisième en restant assis et recourant à des gestes manuels. Les trois élèves suivants miment certains personnages afin de restituer les paroles de ceux-ci en anglais. Les sept 
élèves mentionnés ci-dessus ne se contentent pas d'évoquer le souvenir d'un état empathique: ils souhaitent le revivre. Pour cela, ils ont recours au mime, ce comportement illustrant avec éloquence l'enracinement corporel de l'empathie. Comme le suggère Depraz (2004: 183), le mouvement empathique s'effectue dans la spatialité particulière de notre corps vécu (pour revivre une situation qui relève d'une trame narrative, les enfants interviewés la transportent dans leur espace corporel) et grâce à un couplage temporel des deux corps (pour évoquer les actions passées réalisées par autrui, les enfants les déplacent dans le présent par le biais de leur propre action corporelle).

Pour ce qui est des élèves qui ne produisent pas d'états empathiques in situ mais en évoquent le souvenir, leur discours confirme également «l'ancrage expérientiel » de l'empathie (Ibid. : 187) : en rendant compte de leur rapport aux personnages rencontrés, ils disent «avoir été dans leur peau » (expression employée par trois élèves), «avoir été (le personnage) » (6 élèves), « avoir été dans (le personnage)/dedans » (4 élèves), « avoir été comme (le personnage) » (7 élèves).

En se souvenant de leur projection dans les personnages, onze élèves reprennent les informations explicites des textes abordés, comme dans l'exemple suivant : «quand j'étais flûtiste j'aimais bien quand il fallait jouer et que tous les rats me suivaient $»^{1}$. Neuf élèves vont plus loin en donnant une coloration interprétative personnelle aux textes. Par exemple :

moi j'étais comme le flûtiste ${ }^{2}$ enfin entre les deux (-) le flûtiste et les enfants (-) j'avais peur quand on était enfermés dans la grotte mais j'aime bien avoir peur quand c'est pas pour de vrai et le flûtiste il est parti mais il n'était plus en colère comme la première fois

Le texte de la légende ne donnait pas d'indications explicites sur les émotions des enfants enfermés dans la grotte ni sur celles éprouvées par le flûtiste au moment où il quittait la ville de Hamelin définitivement : l'élève déduit ces détails de son expérience de l'histoire, telle qu'il a pu la vivre en classe. Rappelons que la gestion de l'implicite représente une difficulté de taille pour de nombreux élèves du cycle en question (Paquette Chayer, 1999). Afin de développer cette compétence, les didacticiens de la lecture proposent le plus souvent des pistes de travail liées à l'élaboration de stratégies mentales, en occultant l'implication corporelle de l'élève dans la trame narrative. Cette implication, faisant partie intégrante des approches artistiques pratiquées dans le dispositif, semble favoriser le traitement cognitif des textes : parmi les neuf élèves qui ont fait preuve d'attitudes interprétatives à la fois adaptées et originales, quatre sont des élèves en difficulté qui ne font pas transparaître cette aptitude au cours de séances de lecture classiques.

Dans les exemples présentés jusque-là, les enfants vivent une fusion momentanée complète avec les personnages dont ils parlent. Dans celui qui suit, nous verrons que la projection empathique permet des allers-retours entre l'autre et soi, entre soi et le monde.

TP1 E1 (élève 1) : on se met vraiment dans la peau des personnages (--) moi c'était le petit garçon (-) dans le prince tigre ${ }^{3} \mathrm{j}^{\prime}$ étais le petit garçon qui devenait un tigre (-) ça fait plein d'émotions en même temps (-) ça fait de la tristesse mais aussi de la joie (-) de la tristesse parce qu'il a quitté sa mère tigre et de la joie parce qu'il va retrouver ses vrais parents et après quand il y en a un de chaque côté il sait pas dans quel camp aller

TP2 E2 (élève 2) : il sait pas qui choisir 
TP3 E1 : il se dit bon ben je reviendrai un an chez toi un an chez toi

TP4 E2 : moi je préfère un mois chez les vrais parents après un mois chez la tigresse

TP5 E1 : si ça m'arrivait à moi j'aurais préféré deux semaines chez mes vrais parents et deux semaines chez les tigres ou les lions ou les (...)

TP6 E2 : c'est un peu comme dans les mythes ${ }^{4}$ Médée qui doit choisir entre son père et son fiancé (-) c'est difficile

TP7 E1 : sauf que là elle a choisi vraiment elle peut pas se dédoubler

TP8 E2 : ou alors elle se coupe en deux (rit)

TP9 E1 : après si on se coupe en deux le corps il arrête de vivre (-) il faut que le corps il soit fermé

Dans le premier tour de parole (TP1), E1 témoigne de sa projection empathique dans le personnage du prince tigre par identification (discours à la première personne: «moi c'était le petit garçon", "j'étais le petit garçon ») puis en prenant de la distance (discours à la troisième personne : «il a quitté sa mère », «il va retrouver ses vrais parents », « il sait pas dans quel camp aller »). Cet extrait d'entretien renvoie à l'empathie en tant qu' «... un curieux processus dynamique d'interaction vécue qui exige simultanément d'être soi et un autre, de se vivre soi-même et en même temps d'échapper à ce point de vue égocentré pour adopter un point de vue hétérocentré" (Berthoz, 2004: 254). L'attitude distanciée (discours à la troisième personne) est maintenue (TP2-3) avant un nouveau passage à l'identification (TP4) où E2 se met à la place du prince tigre : «Moi (en tant que prince tigre) je préfere un mois chez les vrais parents (ceux du prince) après un mois chez la tigresse ». E1 continue à s'exprimer à la première personne (TP5) tout en arrêtant de se mettre à la place du personnage : désormais, elle imagine le comportement qu'elle aurait eu dans une situation semblable mais pas identique. On observe ensuite (TP6) une distanciation encore plus marquée: les interlocuteurs s'éloignent, cette fois-ci, non seulement du personnage qui faisait l'objet de la projection mais aussi de leur égo pour conceptualiser le problème du choix. Ils font un parallèle avec le mythe de Médée puis arrivent à la conclusion : la multiplicité expérientielle de la vie est impossible sans l'unité corporelle (TP9). Dans leur langage d'enfants, ils expriment une idée très proche de celle formulée par une des artistes lors de l'entretien semi-dirigé qu'elle avait passé en fin de projet :

Notre corps est la première interface dans notre communication avec l'extérieur (-) c'est magique que ce soit ce corps (-) toujours le même qui puisse nous permettre de vivre tant d'expériences diverses mais finalement fluides et unies (--) c'est le corps qui donne une cohésion à toutes ces différentes expériences.

\subsection{Attitude empathique envers un autre réel : rapport aux pairs et aux artistes}

Si se projeter dans un autre fictif est naturel pour l'enfant (Bettelheim, 1976), la projection dans un autre réel nécessite maturation et apprentissage. L'analyse des entretiens fournit des éléments qui permettent de considérer l'approche pédagogique pratiquée comme contribuant à cette décentration. Etudions-en un exemple. 

après l'autre.

TP1 E3 : là j'ai voulu faire un dessin rythmé ${ }^{5}$ pour Tyger Tyger (-) ah mince j'y arrive plus

(n'arrive plus à redire le texte en suivant les traits de son dessin)

TP2 E4 si on faisait chacune et qu'on le disait

(disent le même texte en faisant deux dessins différents)

TP3 E3 ça fait un gros gribouillage

TP4 E4 mais non ça fait un rythme (-) si quelqu'un qui ne comprend pas l'anglais écoute Tyger il va dire que c'est un gribouillage de sons mais quand on comprend c'est un rythme et une histoire le plaisir d'une expérience phonatoire (TP1). E4 propose à E3 de l'accompagner (TP2). Elles disent en chœur le même texte en s'appuyant chacune sur son geste graphique personnel. A l'issue de cet exercice, E3 change de perspective regardant maintenant son dessin non pas comme support de la récitation mais comme produit pictural qu'elle qualifie de "gribouillage» (TP3). E4 se montre capable d'adopter les deux perspectives qui se profilent dans le discours d'E3 :

- la perspective d'un observateur non-initié qui reste à la superficie de ce qu'il voit en se focalisant sur le résultat et non le processus ;

- la perspective esthétique et cognitive que l'on est en mesure d'emprunter si l'on a partagé l'expérience ayant mené au produit observé.

Cette habileté à adopter et à expliciter différents points de vue (TP4) correspond à la spatialisation du problème de l'empathie qui caractérise l'approche de Berthoz (2004).

Généralement, le rapport empathique de l'enfant aux adultes est encore plus problématique que vis-à-vis des pairs, car il est plus difficile de construire «le cadre de référence interne » (Decety, 2004) de la personne dont la constitution psychique, les centres d'intérêt et les préoccupations sont éloignés des nôtres. Or, l'analyse des entretiens offre huit exemples de ce type d'attitude. Citons-en-un :

TP1 E5 j'ai bien aimé aussi les intermèdes ${ }^{6}$ qu'on a fait avec $\mathrm{W}^{7}(-)$ j'ai bien aimé quand $\mathrm{P}^{8}$ il faisait le papa et $\mathrm{W}$ il courait comme ça (montre) parce qu'il faisait l'enfant (-) pour P c'était pas trop dur je pense (-) nous on voit des adultes tous les jours et on a l'habitude de jouer (-) on peut faire un adulte un animal un personnage des jeux vidéo (-) ce qu'on veut (-) les adultes eux normalement ils ne jouent pas à être un enfant

TP2 E6 oui mais W c'est pas pareil c'est un artiste

TP3 E5 d'accord mais quand même (-) quand tout le monde a rigolé lui il a dû être superconcentré pour ressembler vraiment à un enfant

TP4 E6 c'est comme moi quand je faisais la racine ${ }^{9}(-)$ ma sœur croyait que c'était facile mais moi j'étais superconcentrée

TIPA. Travaux interdisciplinaires sur la parole et le langage, 35 | 2019 

Pacherie (2004), en montrant qu'il comprend :

- le type de l'état affectif d'autrui (une grande concentration);

- l'objet de cet état (l'art du mime) ;

- la raison de cet état (la difficulté à adapter le corps et le psychisme d'adulte à la tâche).

E5 atteint le premier degré de l'empathie par "perception directe» (Ibid.: 167). On pourrait même utiliser le terme de perception/action directe car E5 se souvient des mouvements du danseur sur scène et les reproduit (TP1). Le second et le troisième degré de l'empathie sont atteints par le biais de «l'imagination et de la simulation projective » ( Ibid.) : E5 est en mesure de comprendre l'objet et la raison de l'état affectif du danseur (TP3) puisqu'il possède lui-même une première expérience de pratique de la danse et du théâtre, acquise dans le cadre du dispositif. E6 partage cette compréhension en donnant l'exemple d'un état similaire qu'elle a éprouvé sur scène, lors de la représentation (TP4).

\subsection{Une " conversation (...) conclue harmonieusement $»^{10}$ : exemple d'une expérience aboutie}

D'après Dewey, les expériences tronquées sont caractérisées soit par une « hypertrophie de l'agir " soit par une «hypertrophie de la réceptivité » (2014: 95). Un dialogue impossible peut impliquer différentes typologies de manque d'interaction:

- les deux interlocuteurs affichent "l'hypertrophie de l'agir ", chacun s'employant à monopoliser la parole et refusant de prêter l'oreille aux arguments de l'autre ;

- le comportement d'un interlocuteur relève de l'« hypertrophie de l'agir », celui de l'autre de «l'hypertrophie de la réceptivité»; ce dernier, ayant des difficultés à résister à la pression du premier, adopte une attitude d'opposition muette ou se plie aux opinions qui lui sont imposées.

De nombreux enseignants reconnaîtront ces attitudes comme étant fréquentes dans leurs classes, au cours de débats ou lors de travaux de groupes, et se diront dans la recherche de moyens pour y faire face. Dans le cadre du dispositif expérimenté, nous avons eu recours aux pratiques artistiques pour éduquer les élèves à l'équilibre perception/action car l'art a la particularité de stimuler un tel équilibre, comme nous le savons grâce aux travaux de Dewey. Par ailleurs, le mouvement empathique abouti, c'est-à-dire impliquant les quatre aspects (incorporation, identification, réverbération, détachement) définis par Reik (1948) et Decety (2004), présente également cet équilibre: l'incorporation et l'identification sont des aspects perceptifs, la réverbération et le détachement relèvent, quant à eux, de l'action.

En analysant l'extrait de corpus suivant, nous verrons comment s'agencent les éléments perceptifs et actifs dans les interventions de trois élèves-interlocuteurs.

TP1 E7 là c'est le pont là c'est les barrières ça c'est le soleil qui brille (-) la sorcière va traverser (-) ça c'est l'étang ça c'est le vent des arbres (--) en fait la tigresse elle traverse ce pont aussi quand elle va de la forêt au village

TP2 E8 le flûtiste quand il arrive dans la ville il le traverse ce pont lui aussi (pointe l'élément du dessin concerné)

TIPA. Travaux interdisciplinaires sur la parole et le langage, 35 | 2019 
TP3 E9 et dans nos histoires des couleurs le bleu on peut dire qu'il traverse le pont calmement (-) c'est un endroit calme et le rouge il saute sur les nénuphars boum boum et après c'est le vert qui vient le calmer (procède à une gestuelle illustrative : les doigts qui « marchent », « sautent »)

TP4 E8 le vert des arbres (pointe les arbres)

TP5 E9 et après il y a le jaune qui arrive et il fait boing boing (-) il énerve tout le monde

TP6 E8 on aurait pu danser ça avec $\mathrm{M}$ et $\mathrm{W}^{11}$

TP7 E7 l'artiste avec son pinceau magique c'est peut-être lui qui a peint le pont et comme c'était le pinceau magique c'est devenu réel (-) il peint le pont sur une feuille dans la classe et après le pont il devient réel et il est là dans la classe

(Les trois enfants se lèvent et font semblant de traverser le pont. Puis l'échange s'oriente vers un thème différent).

Chaque tour de parole sera analysé en termes de processus perceptifs (l'enfant se laisse imprégner par les émotions et la pensée de son interlocuteur) et (ré)actifs (l'enfant s'insère dans la proposition de son interlocuteur en développant celle-ci et, parfois, en lui accordant une nouvelle tournure).

\begin{tabular}{|c|c|c|c|}
\hline TP & Elève & Perception & Action \\
\hline 1 & E7 & $\begin{array}{l}\text { Pour répondre à la } \\
\text { demande de dessiner son } \\
\text { année de CE1, l'enfant } \\
\text { retrouve dans sa mémoire } \\
\text { sensorielle le moment qui } \\
\text { l'a particulièrement } \\
\text { marqué : il s'agit d'une } \\
\text { séquence interdisciplinaire } \\
\text { autour de l'œuvre de } \\
\text { Monet. }\end{array}$ & $\begin{array}{l}\text { E7 reconstitue et commente une des versions des } \\
\text { Nymphéas de Monet. La mention d'une sorcière (ou, plus } \\
\text { exactement, d'une fée, terme oublié par l'élève) renvoie } \\
\text { à la trame du conte conducteur qui avait permis } \\
\text { d'initier la classe à la vision impressionniste du monde. } \\
\text { Pour E7, le paysage qu'il dessine n'est pas un fragment } \\
\text { de l'expérience vécue au cours de l'année mais une } \\
\text { représentation symbolique de celle-ci. Ainsi, il fait } \\
\text { emprunter à la tigresse, personnage de l'album Le } \\
\text { prince tigre, le même pont qu'à la fée/sorcière. Cette } \\
\text { association élaborée par E7 n'est pas aléatoire - le pont } \\
\text { sert, dans les deux cas, à relier deux espaces } \\
\text { métaphoriques opposés: ceux du monde réel et du } \\
\text { monde magique, dans le cas de la fée/sorcière; ceux du } \\
\text { monde sauvage et du monde civilisé, dans le cas de la } \\
\text { tigresse. }\end{array}$ \\
\hline
\end{tabular}




\begin{tabular}{|c|c|c|c|}
\hline 2 & E8 & $\begin{array}{l}\text { E8 se relie au processus à la } \\
\text { fois affectif et cognitif } \\
\text { entamé par son camarade. }\end{array}$ & $\begin{array}{l}\text { E8 propose une suite logique de la chaîne associative } \\
\text { le flûtiste (personnage de la légende Le joueur de flûte de } \\
\text { Hamelin) arrive dans la ville par ce même pont. Notons } \\
\text { que l'action de traverser un pont n'est pas mentionnée } \\
\text { dans le texte de la légende, mais reste plausible, que ce } \\
\text { soit sur le plan réaliste (une rivière coule dans la ville } \\
\text { en question) ou symbolique (à l'instar de la fée qui } \\
\text { apparaît dans le récit d'E7, le flûtiste se déplace entre } \\
\text { le monde magique et le monde réel). }\end{array}$ \\
\hline $\begin{array}{l}3 \\
\text { et }\end{array}$ & E9 & $\begin{array}{l}\text { E9 adhère à la proposition } \\
\text { d'appuyer l'échange sur le } \\
\text { dessin d'E7. }\end{array}$ & $\begin{array}{l}\text { E9 dévie la conversation de la symbolique du passage, } \\
\text { axée sur un seul élément du dessin (le pont), en } \\
\text { suggérant de se concentrer plutôt sur la dynamique } \\
\text { des mouvements (calme ou nerveux, fluide ou brusque) } \\
\text { associés aux couleurs primaires, et de s'intéresser à } \\
\text { d'autres éléments du dessin (nénuphars). Il se réfère à } \\
\text { l'expérience picturale/verbale/dansée vécue autour de } \\
\text { l'œuvre de Delaunay. }\end{array}$ \\
\hline 4 & E8 & $\begin{array}{l}\text { E8 accueille favorablement } \\
\text { l'entrée symbolique et } \\
\text { spatiale suggérée par E9. }\end{array}$ & $\begin{array}{l}\text { E8 s'insère dans le discours d'E9 pour insister sur le } \\
\text { lien du récit avec l'espace élargi du dessin : aux } \\
\text { mentions du pont et des nénuphars, elle ajoute celle } \\
\text { des arbres. }\end{array}$ \\
\hline 6 & E8 & $\begin{array}{l}\text { E8 } \text { se laisse imprégner par } \\
\text { la coloration kinesthésique } \\
\text { et émotionnelle de } \\
\text { l'intervention de son } \\
\text { camarade. }\end{array}$ & $\begin{array}{l}\text { Forte de l'expérience de travail avec les danseurs, E8 } \\
\text { est consciente que la production narrative de son } \\
\text { camarade aurait pu faire l'objet d'un exercice } \\
\text { d'improvisation dansée. }\end{array}$ \\
\hline 7 & E7 & $\begin{array}{l}\text { La référence à la danse } \\
\text { mène E7 à retrouver le } \\
\text { sentiment qu'il a exprimé } \\
\text { au sujet de la danse un peu } \\
\text { plus tôt, au cours de ce } \\
\text { même entretien: "j'aime la } \\
\text { danse parce que ce qu'on y } \\
\text { fait est vrai». }\end{array}$ & $\begin{array}{l}\text { Transporté par l'authenticité que lui inspire l'approche } \\
\text { globalement corporelle pratiquée en danse, E7 évoque } \\
\text { l'artiste, personnage du conte Le pinceau magique, dont } \\
\text { les œuvres se transforment systématiquement en } \\
\text { objets ou êtres authentiques (réels). Revenant à sa } \\
\text { focalisation initiale sur le pont, E7 conduit ses } \\
\text { interlocuteurs à quitter l'espace de son dessin et à } \\
\text { imaginer un pont réel posé en plein milieu de la salle } \\
\text { de classe. }\end{array}$ \\
\hline
\end{tabular}

L'analyse de l'échange met en lumière l'équilibre perception/action dans le discours des enfants et permet d'y déceler l'ensemble des caractéristiques d'une communication empathique: "une réponse affective envers autrui qui implique (mais pas toujours) un partage de son état émotionnel ; la capacité cognitive de prendre la perspective subjective de l'autre personne » (Decety, 2004:57), «la distinction entre soi et l'autre, et ce en parallèle avec un éventuel partage affectif » (Ibid. : 54). L'analyse donne également à voir quatre aspects d'un acte empathique abouti (Reik, 1948 ; Decety, 2004) :

- l'identification de chaque enfant à son interlocuteur par le biais du dessin d'E7, qui sert d'objet transitionnel (TP2-6) ; 
- la réverbération, ou, autrement dit, une réponse en écho à l'interlocuteur (poursuite de la chaîne associative, comme dans le TP2, ou encore de l'élargissement de l'ancrage topographique de l'échange, comme dans le TP4);

- l'incorporation (cet aspect est mêlé aux deux précédents et se traduit par la gestuelle illustrative qui anime la parole, comme dans les TP2, 3, 4, 7);

- le détachement (l'interlocuteur modifie la direction de l'échange tout en maintenant le lien avec ce qui s'est dit auparavant, comme dans les TP3 et 7).

\section{Résultats et conclusion}

L'analyse des entretiens conduits avec les élèves, croisée avec l'analyse des entretiens effectués auprès des artistes et de mon propre journal de bord, tenu tout au long de l'année d'expérimentation, ont permis d'aboutir aux résultats suivants :

- les élèves ont considérablement progressé dans le développement de la compétence d'empathie, en se transformant d'une classe où, en début d'année scolaire, des comportements égocentrés et des situations de conflit étaient fréquents, en une classe où l'écoute et la bienveillance ont progressivement pris le dessus;

- les élèves ont fait preuve de comportements empathiques complexes englobant tous les degrés (Pacherie, 2004) et l'ensemble des aspects (Reik, 1948 ; Decety, 2004) de l'empathie ;

- tout en développant des conduites pro-sociales au travers de la compréhension empathique de l'autre, les enfants ont appris à mieux cerner leur « soi » et à avoir un regard réflexif sur le monde qui les entoure ;

- ils se sont montrés capables de mieux conscientiser, contrôler, canaliser et exprimer leurs émotions à l'aide des langages verbal, dansé, pictural, musical ;

- ils ont développé l'habileté à déceler la dimension implicite dans les comportements des personnes qu'ils côtoient ainsi que dans les œuvres d'art qu'ils étudient.

Des leçons de morale ne sont pas susceptibles d'engendrer une communication empathique ; pour qu'elle advienne, sont nécessaires « une similarité d'expérience, une communauté de culture » (Decety, 2004: 58) bâties tout au long du projet dont il est question dans cet article.

Quelles que soient les attitudes empathiques manifestées, elles résultent d'une expérience à la fois commune (propre à la communauté-classe) et singulière (propre à chaque participant) qu'illustre parfaitement le témoignage d'un élève : « on faisait tous des choses différentes mais on était ensemble ». C'est au travers d'un processus créatif partagé que chacun a pu s'immerger dans le monde subjectif d'autrui et en ressortir pour retrouver un soi distinct, stimulé et enrichi par la rencontre. L'équilibre perception/action a servi de terrain à une meilleure compréhension de l'autre que l'on intègre « dans le flux de son vécu (...) et non pas dans une simple théorie de l'esprit de l'autre » (Berthoz, $2004: 266$ ).

Les résultats obtenus dans le cadre de cette recherche qualitative ne peuvent être généralisés étant donné la taille réduite de l'échantillon (une classe/une enseignantechercheuse/un groupe d'artistes) et un contexte pédagogique exceptionnel (dévouement de tous les adultes engagés dans le projet, disponibilité d'un budget permettant de rémunérer les artistes, soutien de l'inspection académique et de la municipalité). D'autres projets de recherche seraient nécessaires pour vérifier ces résultats et, au cas de leur confirmation, élaborer des outils de formation à ce type d'approche pédagogique. 

qu'à long terme, il est urgent de se pencher d'ores et déjà sur la question de l'éducation à l'empathie de manière générale. Les textes officiels nous y obligent à l'heure où le « respect d'autrui » est ajouté aux fondamentaux traditionnels consistant à savoir « lire, écrire et compter » (Ministère de l'Education nationale, 2018). Or, comment promouvoir le respect de l'autre sans s'intéresser à ses émotions et à sa corporéité qui se trouvent à la base de l'empathie? Quant aux fondamentaux traditionnels, des études scientifiques comme celle de M.-H. Immordino-Yang (2016) montrent qu'il n'est plus possible de les assurer aujourd'hui en pratiquant des méthodes pédagogiques qui privilégient l'esprit en ignorant le corps, qui s'intéressent au développement cognitif de l'individu sans tenir compte de son évolution dans l'environnement social.

\section{BIBLIOGRAPHIE}

Aden, J. (2012) La médiation linguistique au fondement du sens partagé : vers un paradigme de l'énaction en didactique des langues, Etudes de linguistique appliquée, 167, p. 267-284.

Aden, J. (2017) Langues et langage dans un paradigme énactif, Recherches en didactique des langues et des cultures [en ligne], 14-1 | 2017. Disponible sur : http://rdlc.revues.org/1085 (consulté le 28 novembre 2018).

Berthoz, A. (2004) Physiologie de changement de point de vue, in Berthoz, A. \& G. Jorland, (dir.) L'Empathie, Paris: Odile Jacob, p. 251-275

Bettelheim, B. (1976) Psychanalyse des contes de fées, Paris: Robert Laffont.

Catheline, N. (2015) Le harcèlement scolaire, Paris: PUF (Que sais-je ?).

Damasio, A. R. (2002) Le Sentiment même de soi, Paris: Odile Jacob.

Decety, J. (2004) L'empathie est-elle une simulation mentale de la subjectivité d'autrui ?, in Berthoz, A. \& G. Jorland (dir.), L'Empathie, Paris: Odile Jacob, p.53-88.

Depraz, N. (2004) Empathie et compassion, in Berthoz, A. \& G. Jorland (dir.) L'Empathie, Paris: Odile Jacob, p. 183-199.

Dewey, J. (1934/2014) L'art comme expérience, Paris: Folio Essais.

Eschenauer, S. \& A.-M. Voise (2017) Partager les langues et les cultures au cycle 3, Les langues modernes, $2 / 2017$.

Immordino-Yang, M.-H. (2016) Emotions, Learning, and the Brain, New-York/London: Norton.

Lecoq, J. (1997) Le corps poétique, Arles: Actes Sud.

Luquet, G.H. (1984) Le dessin enfantin, Neuchâtel: Delacheux \& Niestlé.

Maturana, H. \& F. Varela (1998) The Tree of Knowledge. The Biological Roots of Human Understanding, Boston/ Londres: Shambhala.

Ministère de l'Education nationale (2015) Socle commun de connaissances, de compétences et de culture. Bulletin officiel $n^{\circ} 17$ du 23 avril 2015. [En ligne] Disponible sur : http:// 
cache.media.education.gouv.fr/file/17/45/6/

Socle_commun_de_connaissances,_de_competences_et_de_culture_415456.pdf (consulté le 22 février 2019).

Ministère de l'Education nationale (2018) Renforcer les fondamentaux : la lettre du ministre de l'Education nationale aux professeurs. 26 avril 2018. [en ligne] Disponible sur : http:// www.education.gouv.fr/cid129676/renforcer-les-fondamentaux-la-lettre-du-ministre-de-leducation-nationale-aux-professeurs.html (consulté le 25 février 2019).

Pacherie, E. (2004) L'empathie et ses degrés, in Berthoz, A. \& Jorland, G. (dir.) L'Empathie, Paris: Odile Jacob, p. 149-181.

Paquette Chayer, L. (dir.) (1999) Compréhension de lecture, Montréal: Editions Chenelière.

Potapushkina-Delfosse, M. (2014) Débuter l'apprentissage de l'anglais par le geste : de la démarche d'enseignement aux stratégies mnésiques des élèves, Les Cahiers du CRINI, Languages in Motion/Langues en mouvement [en ligne]. Disponible sur : http://www.crini.univnantes.fr/1403000125802/0/fiche_pagelibre/\&RH=1402999468883 (consulté le 25 février 2019).

Potapushkina-Delfosse, M. (2016) Vers une méthodologie d'évaluation holistique pour une approche d'enseignement de l'anglais à l'école primaire, LEND - Lingua e Nuova Didattica, 2/2016, Approcci teatrali nella didattica delle lingue : parola, corpo, creazione, p.125-135.

Potapushkina-Delfosse, M. (2018) Se mouvoir et s'émouvoir pour apprendre une langue vivante à l'école, in Berdal-Masuy, F. (dir.) Emotissage, les émotions pour apprendre les langues, Louvain: UCL Presses Universitaires, p. 105-115.

Raby, F. (2008) Entre faux débats et vraies distinctions. Réflexions sur les méthodes de recherches empiriques en didactique de l'anglais, Les Cahiers de l'ACEDLE [en ligne]. Disponible sur : http:// ardaarda.canablog.com/archives/2010/10/09/19280861.html (consulté le 26 février 2019).

Reik, T. (1948) Listening with the third ear; the inner experience of a psychoanalyst, New York: Farrar, Straus \& Co.

Siekkinen, M., Pakarinen, E., Lerkkanen, M.-K., Poikkeus, A.-M., Salminen, J., Poskiparta, E. \& J.-E. Nurmi (2013) Social competence among 6-year-old children and classroom instructional support and teacher stress, Early Education and Development, 24, p. 877-897.

Varela, F. (1994) Né pour organiser (film documentaire) in H. Trocmé-Fabre (dir.), ENS Fontenay/St Cloud: Université de la Rochelle.

Varela, F., Thompson, E. \& E. Rosch (1993) L'inscription corporelle de l'esprit, Paris: Seuil.

Vermersch, P. (1994/2010) L'entretien d'explicitation, Paris: ESF Editeur.

\section{NOTES}

1. La transcription est réalisée à partir des enregistrements audio des entretiens. Voici quelques éléments du code utilisé : pas de matérialisation de la ponctuation; respect des majuscules uniquement pour les noms propres ; (-) : pause brève ; (--) : pause prolongée, (...) : interruption. Les maladresses de langage ne sont pas corrigées.

2. Dans cette citation ainsi que dans la précédente, les locuteurs se réfèrent à la légende Le joueur de flûte de Hamelin.

3. Le locuteur fait référence à l'album Le prince tigre.

4. Au cours de cette année scolaire, les élèves ont découvert la mythologie grecque grâce au rituel de lecture magistrale quotidienne qui marquait chaque fin de journée de classe. 
5. La seconde phase du dispositif a permis aux élèves d'apprendre le texte adapté du Joueur de flûte de Hamelin à l'aide d'une gestuelle picturale à fonction mnésique (« dessin rythmé »), créée par la classe sous mon guidage. La locutrice a réalisé un dessin similaire pour le poème The Tyger. Elle essaie de redire le texte en repassant son dessin au doigt mais n'y parvient pas.

6. Elément semi-improvisé de la représentation dansée finale

7. Un des danseurs ayant participé à la dernière phase du dispositif

8. Un élève

9. Elément de la représentation dansée finale

10. Dewey $(2014: 81)$

11. Artistes

\section{RÉSUMÉS}

Cet article présente un dispositif expérientiel associant l'apprentissage d'une langue vivante (anglais) à plusieurs pratiques artistiques (arts visuels, danse, poésie, musique), développé à partir de la théorie de "l'art comme expérience » de Dewey (1934/2014) et mis en œuvre au cours d'une année scolaire, au sein d'une classe de CE1, dans le cadre d'une recherche action. Apprendre une langue n'implique pas uniquement le développement de compétences "techniques» (phonologiques, lexicales, grammaticales) mais aussi, et avant tout, la construction de l'habileté à langager (Maturana, Varela, 1994, Aden, 2017), c'est-à-dire, se relier à soi et à autrui. Les entretiens conduits avec les élèves en fin d'année, selon la méthodologie proche de celle d'explicitation, adaptée à l'âge du public et aux particularités de l'environnement scolaire, ont fait l'objet de l'analyse qui a permis l'identification d'éléments variés se rapportant aux états d'empathie, éprouvés par les élèves. Divers comportements empathiques stimulés par l'approche didactique, mettant en synergie le cognitif et le sensible, sont explicités avec l'appui sur des travaux de Berthoz, Jorland et al. (2004).

This paper is dedicated to an experiential approach to teaching/learning foreign languages, associated with various artistic practices (painting/drawing, dance, singing, poetry), created under the influence of John Dewey's theory of "art as experience" (1934/2014).

Before talking about an experiential approach, it is important to clarify the concept of experience as Dewey defined it: "the interaction of live creature and environing conditions (that) is involved in the very process of living" (1934/2014: 80). He distinguished experience (qualified by distraction, dispersion, usually stopped before attaining its aim) from an experience (characterized by fulfillment and harmony). An experience can result from activities of many kinds but is most spontaneously achieved in the field of arts. Dewey explains this fact by the simultaneity of perception and action, which is typical both for artistic processes and the process he calls an experience.

It is commonly admitted that being in contact with arts (as a listener, reader, etc.) implies perceptive behavior and being producing artwork involves an active position. By contrast with that widespread view, Dewey notes the necessary co-presence of perception and action in both cases. Being an artist and being in contact with art require co-creation, which is a deeply empathetic attitude:

- the person who is going through an esthetic experience enters into empathy with the author or the characters of the artwork he/she is exploring; 
- while creating, the artist empathizes with "an indispensable partner" that is always present "even when the artist works in solitude".

Given those considerations, it is not surprising to find that empathy and art share a common definition: "art is a mode of knowledge" (Dewey, 1934/2014 : 465) / "empathy is a mode of knowledge" (Pacherie, 2004 : 150). Art and empathy as cognitive (and not only affective) tools seem appropriate to be chosen as the basis of a learning process that aims to lead pupils to a meaningful holistic experience.

However, one may ask what link can be found between art/empathy and languages. Each new language is learned through the already mastered modes of expression. Art that is, according to Dewey, "the most universal and freest form of communication" (Ibid. : 440) is confined in museums in the adult world but is a part of children's everyday life : they use artistic languages (fictional narration, drawing, singing, etc.) as naturally as they speak the verbal language in order to communicate. This is the reason that made me try to use artistic languages as media to foreign language learning. In my research, I have shown, over the last years, the positive impact of such an approach on the development of pupils' linguistic abilities (pronunciation, memorization of vocabulary and grammatical structures). As for this paper, it focuses on transversal empathy skills. How and to what extent do pupils develop them when language learning is accompanied by artistic practices? In order to answer this question, I conducted a one-year experimental project within a class of 26 7-8-year-old pupils, in a primary school, near Paris. The project comprised four stages of gestural/danced, pictorial, verbal (in French and English) creative activities around:

- Sonia Delaunay's paintings;

- The Pied Piper of Hamelin (German legend);

- The Magic Paintbrush (Chinese fairy-tale);

- William Blake's poem The Tyger.

The fourth stage was conducted in cooperation with Mobilis Immobilis dance company and concluded with a performance produced by the class under the guidance of the artists.

At the end of the year, all the pupils were interviewed following the method of explicitation interview (Vermersch, 2010) adapted to the interviewees' age as well as to the specificities of the school context. Each child was invited to draw his/her "art-language" school year and then to talk about it, explaining the drawing as a start. During the interview, each participant expressed him/herself, listened to other participants within the group, and interacted with them. The analysis of those interactions, based on the theoretical background provided by A. Berthoz (neuropsychology), J. Decety (developmental and affective neuroscience), N. Depraz and E. Pacherie (philosophy) made it possible to identify various types and degrees of empathy shown by children towards fictional characters, other pupils and dancers who worked with the class during the last stage of the project.

The most frequent empathetic attitude found in the analyzed interactions is directed towards fictional characters. It is not surprising, since children of this age need to be guided by fiction, for example by fairy-tales (Bettelheim, 1976), that provide them with symbolic answers to problems they encounter on their way towards building identity and social relationships.

While having empathic feeling towards a fictional other is natural for a child, empathizing with a real other requires maturity and an adequate emotional environment, if we mean by empathy "an affective response to an other person, that implies (but not always) sharing this person's emotional state; a cognitive ability to take this person's subjective perspective" (Decety, 2004 : 57), "distinction between the self and the other in parallel with a possible affective sharing" (Ibid. : 54). Berthoz (2004 : 254) refers to Piaget's theory according to which, before the age of 7-8, "the child is a prisoner of his/her mental schemas, feeling like a person shut in a labyrinth with the only path leading to the only exit, the only possible point of view (...) on the world". However, from the age of $7-8$, the child starts transforming this egocentric position into a more allocentric 
one, the quality and speed of this change depending on many factors, education being one of them. The analysis of the interviews allows to consider the implemented pedagogic approach as contributing to the children' decentering.

The detailed interpretation of the selected interview excerpts corresponding to various types and degrees of empathetic attitudes shows that, unlike the adults for whom the "mental simulation" (Berthoz, Jorland, 2004) of someone else's subjectivity is sufficient to access an empathetic state, the child, most of the time, needs to go through a physical simulation which is made possible and sensible thanks to artistic practices integrated into the project.

Empathy results from "a similarity of experience, a community of culture" (Decety, 2004 : 58). The project provided a framework for such an experience, common (belonging to the whole class) and, at the same time, singular (conducted, perceived, understood in an individual manner by everyone). Throughout the creative process that unified pupils, everyone could become immersed in the subjective world of the others, then go back to his/her singularity enriched, stimulated by the encounter, in a cyclic harmonious way.

\section{INDEX}

Mots-clés : expérience, langages, empathie, perception/action, pratiques artistiques

Keywords : experience, languages, empathy, perception/action, artistic practices

\section{AUTEUR}

\section{MARIE POTAPUSHKINA-DELFOSSE}

Université de Rouen Normandie, ERIAC EA 4705, ESPE

potapushkina.m@gmail.com 